\title{
A New FRET-Based Sensitive DNA Sensor for Medical Diagnostics using PNA Probe and Water-Soluble Blue Light Emitting Polymer
}

\author{
Nidhi Mathur, Anamika Aneja, P. K. Bhatnagar, and P. C. Mathur \\ Department of Electronic Science, University of Delhi South Campus, Benito Juarez Road, New Delhi-110021, India \\ Correspondence should be addressed to Anamika Aneja, anamica_30@yahoo.co.in
}

Received 2 January 2008; Revised 4 April 2008; Accepted 3 June 2008

Recommended by Mike McShane

A reliable, fast, and low-cost biosensor for medical diagnostics using DNA sequence detection has been developed and tested for the detection of the bacterium "Bacillus anthracis." In this sensor, Poly [9,9-di (6,6' - N, N' trimethylammonium) hexylfluorenyl-2, 7-diyl)-alt-co- (1,4-phenylene)] dibromide salt (PFP) has been taken as cationic conjugated polymer (CCP) and PNA attached with fluorescein dye (PNAC*) as a probe. The basic principle of this sensor is that when a PAC* probe is hybridized with a single strand DNA (ssDNA) having complementary sequence, Forster resonance energy transfer (FRET) may take place from PFP to the PNAC*/DNA complex. If the FRET is efficient, the photoluminescence from the PFP will be highly quenched and that from PNAC* will be enhanced. On the other hand, if the DNA sequence is noncomplementary to PNA, FRET will not occur.

Copyright (C) 2008 Nidhi Mathur et al. This is an open access article distributed under the Creative Commons Attribution License, which permits unrestricted use, distribution, and reproduction in any medium, provided the original work is properly cited.

\section{INTRODUCTION}

Conventional methods of medical diagnostics are generally very expensive and time consuming and are mostly based on antibody detection of the infected person. In these methods, the structure of the antibodies and the antigens they bind to have to be first determined. Sometimes the cost of diagnostics may become even higher than that of the treatment, which makes the need for fast, cheaper, and reliable medical diagnostic techniques indispensable. DNA sequence detection is a topic of major scientific interest. Its application areas include clinical diagnostics, single nucleotide polymorphism (SNP) genotyping, environmental studies, antiterrorism, and forensic analysis [1]. This has motivated the development of novel DNA sensors with optical [2-6], acoustic [7,8], or electronic [9-13] readout. Among these, optical (fluorescent) detection methods have made their place because of their high sensitivity, selectivity, and ease of operation [14]. Homogeneous DNA hybridization assays based on Forster resonance energy transfer (FRET) between donor and acceptor molecules are attractive due to their simplicity of operation and the use of standard optical equipment. Conjugated polymers (CPs) possess a unique combination of optoelectronic properties that have found use in a variety of areas [15]. In the present biosensor application, CPs serve as light-harvesting molecules. The CPs exhibit efficient energy migration along their delocalized backbone and the collected energy can be efficiently transferred to acceptors via FRET [16-19]. The chemical structures of conjugated polymers offer several advantages as the responsive basis for chemical and biological detection schemes based on optical methods. CPs may be viewed as a collection of short, conjugated (oligomeric) units kept in close proximity by virtue of the polymer backbone. Their structure allows effective electronic coupling and hence fast intra- and interchain energy transfer $[20,21]$.

In this paper, we report the method of preparation, operation, and application of a biosensor that provides fast, sensitive, and selective detection of DNA hybridization by using water-soluble cationic conjugated polymer (CCP) and PNA probe. The three components of the assay are a blue light emitting cationic conjugated polymer, that is, polyfluorenephenylene (PFP), a peptide nucleic acid probe labeled with fluorescent dye ( $\mathrm{PNAC}^{*}$ ), $\mathrm{C}^{*}$ being fluorescein dye, and the negatively charged target single strand DNA (ssDNA), which may be complementary (cDNA) or noncomplementary (nDNA) to the PNA sequence. FRET between the donor polymer and the acceptor PNAC* $^{*}$ is used to detect the 


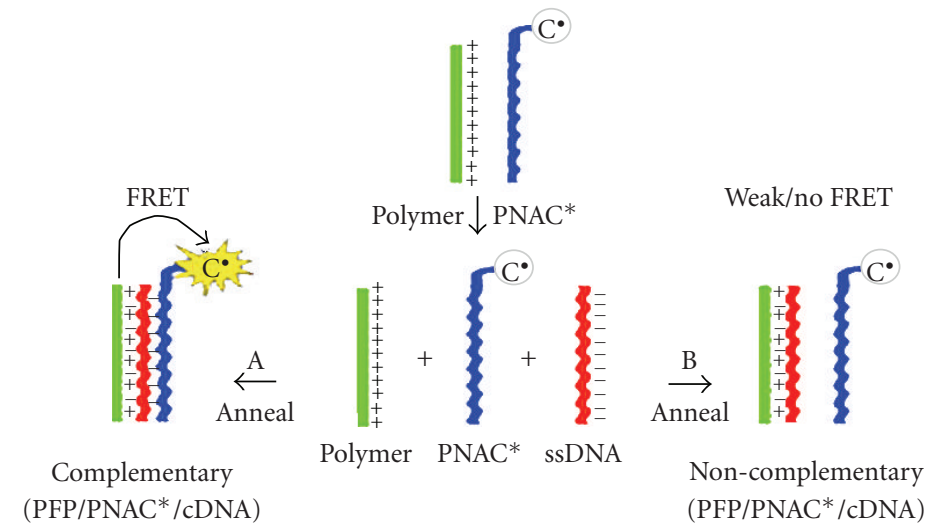

FiguRE 1: Schematic representation of a sensor using polymer and PNAC* probe to detect complementary DNA sequence [28].

presence of specific ssDNA sequence when the sequence of DNA and PNA is complimentary to each other. PNA/DNA complexes hybridize more quickly and are tighter and more specific than analogous DNA/DNA complexes [22]. This is because of the fact that there is columbic repulsion between negatively charged DNA strands, which is absent in the case of negatively charged DNA and neutral PNA. However, PNA complexes are thus more thermally stable and, by virtue of their backbone, less susceptible to biological degradation by nucleases, proteases, and peptidases [23, 24]. Additionally, their general insensitivity to ionic strength and $\mathrm{pH}$ during hybridization provides a wider platform for DNA detection.

When PFP is supplied with sufficient quantum energy, it absorbs photons and reaches an electronically excited state (excited singlet state) and emits blue PL. Once formed, the excited singlet state immediately loses its excess energy and interacts with the sourroundings. The exciton can lose its energy in a number of ways viz, fluorescence, intersystem crossing, photobleaching, collisional interaction with the quenching species, and radiationless transfer of energy (FRET) [25] to a nearby molecule leading to the excitation of the neighbour. In this case, exciton loses its energy (within few ps) by the radiationless transfer of energy to the nearby acceptor PNAC* molecule leading to its excitation which results in the emission of green light from the acceptor (fluorescent dye). In the composite solution, hybridization of the neutral PNA probe to the negatively charged ssDNA significantly creates an electrostatic attraction between the negative PNAC*/DNA complex and the positive PFP, thus brings the fluorophore and the polymer sufficiently close to allow efficient FRET, that is, well within the Forsters critical radius. The time scale in which energy is transferred from donor to acceptor depends on the distance between them. When this distance is close to Forster critical radius, the energy transfer occurs within femtoseconds [26]. On the other hand, the excited state decay of donor PFP occurs exponentially with an average lifetime of $\approx 90 \mathrm{ps}(\approx 30 \mathrm{ps}$ in presence of the acceptor) [27]. The electrostatic energy is, therefore, transferred from donor to acceptor before the former can emit its photoluminescence (PL). The PL emitted by the donor will be quenched significantly provided the distance between the two is within the Forster critical radius.

When the PFP is added to negatively charged PNAC*/ DNA complex, it binds only to the regions where there is a complementation between DNA and PNAC*. The optical amplification due to FRET is the result of optically exciting the polymer (PFP) which under favourable conditions (appropriate distance between donor and acceptor in $\mathrm{nm}$ ) transfers energy to the dye, which emits amplified characteristic green light. On the other hand, for noncomplementary ssDNA, no transfer of energy takes place and hence there is no or very weak emission from the acceptor. A detailed schematic diagram of the sensor is shown in Figure 1.

\section{EXPERIMENTAL}

\subsection{Materials}

For developing a typical sensor for Bacillus anthracis detection, we have selected a blue light emitting, water-soluble, cationic conjugated polymer (PFP), a known PNA probe corresponding to a segment of Anthrax (Bacillus anthracis) attached to the fluorescent dye (fluorescein), two DNA primers, one complementary and the other noncomplementary to the PNA sequence. The light-emitting watersoluble cationic conjugated polymer was purchased from American Dye Source, Inc., Canada. The PNA probe for Bacillus anthracis was purchased from Panagene, Korea. A 20-mer strand labeled with Fluorescein dye corresponding to the following sequence was used:

\section{PNA 20 mer Flu-GTAAATGGTGTTAGGGTTGC $\mathrm{N}$-term C-term.}

For the PNA sequence, "N" represents the free amino group, corresponding to the N-terminal of a peptide, and " $\mathrm{C}$ " is the amidated carboxyl terminal, corresponding to the C-terminal of a peptide. The PNA is known to display hydrophobic interactions, leading to aggregation at high concentrations, suggesting that the experiments must be carried out at very low concentrations $\left(<10^{-5} \mathrm{M}\right)$. The 


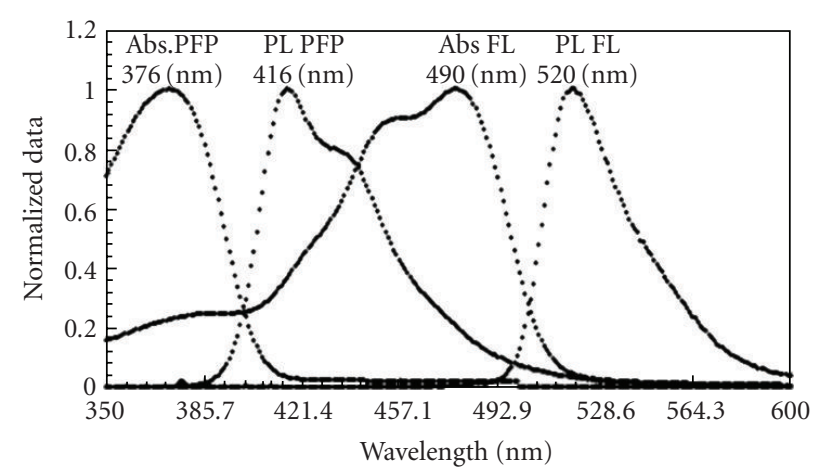

Figure 2: Absorption and emission spectra of PFP (at $10^{-7} \mathrm{M}$ ) and that of PNAC* (at $\left.10^{-8} \mathrm{M}\right)$.

ssDNA corresponding to the following sequences were obtained from Bangalore Genei, India:

ssDNA (Primer sequence $5^{\prime}$ to $3^{\prime}$ ) 20 mer

GCAACCCTAACACCATTTAC (cDNA),

ssDNA (Primer sequence $5^{\prime}$ to $3^{\prime}$ ) 20 mer

GACTCAATGGCGTTAGACTG (nDNA).

\subsection{Spectroscopic measurements}

The solutions of $\mathrm{PNAC}^{*}$ and DNA were prepared in deionized water obtained from TKA water filtration system. For PFP, the solvent mixture of deionized water/ethanol $(1: 1 \mathrm{v} / \mathrm{v})$ was used. The fluorescence measurements of PFP and PNAC* ${ }^{*}$ were made using Shimadzu Spectrofluorophotometer model RF-5301-PC equipped with a xenon lamp excitation source and absorption spectra were observed using Shimadzu UV Visible Spectrophotometer model UV2450 for different molar concentrations. The spectra were recorded in the range $350 \mathrm{~nm}$ to $700 \mathrm{~nm}$. The spectral overlap between the emission spectrum of the donor PFP and the absorption spectrum of the acceptor PNAC* is shown in Figure 2. PNAC* ${ }^{*}$ was used in the experiment at $\mathrm{pH} 5.5$ by using $0.1 \mathrm{~N}$ HCL and deionized water. After optimizing the concentrations of the donor $\left(2.3 \times 10^{-7} \mathrm{M}\right)$ and acceptor $\left(2.5 \times 10^{-8} \mathrm{M}\right)$, the PNA probe $\left(\mathrm{pH}=5.5\right.$ at $\left.10^{-8} \mathrm{M}\right)$ was annealed at $70^{\circ} \mathrm{C}$, both with its complementary ssDNA and the noncomplementary ssDNA $\left(10^{-8} \mathrm{M}\right)$ separately. Annealing was done for about 35-40 minutes in order to improve the hybridization between the ssDNA and PNAC* [28]. The solutions were allowed to cool slowly up to the room temperature and PFP was added in small steps of $25 \mu \mathrm{L}$ of $2.3 \times 10^{-7} \mathrm{M}$ to the above solution, that is, $150 \mu \mathrm{L}$ each PNAC* ${ }^{*}$ and ssDNA in equimolar concentrations of $2.5 \times 10^{-8} \mathrm{M}$. Samples were placed in a vortex mixer between each fluorescence measurement (after each addition of the polymer) to mix the components well. The resulting fluorescence emission spectra were compared and it was observed that the enhancement in the Fluorescein emission was maximum, that is, FRET became saturated when the ratio of the acceptor (PNAC* ${ }^{*}$ ) to the donor (PFP) was about 1:1 after which no change is observed in the fluorescence intensity obtained from PNAC* as shown in Figure 3.

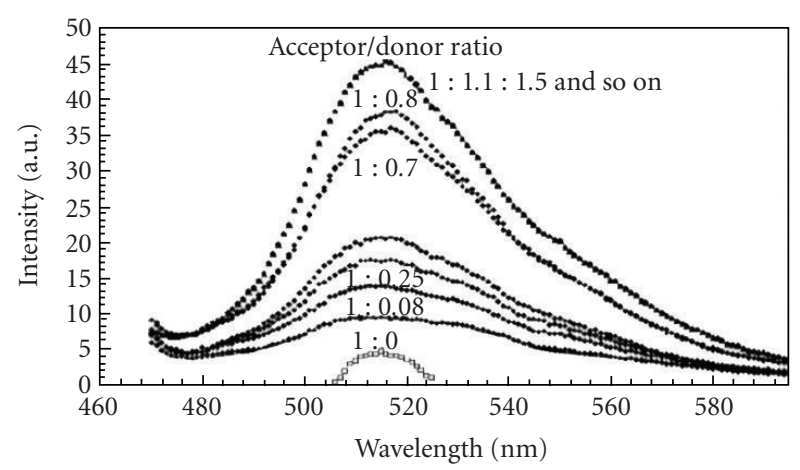

FIGURE 3: Variation of PL emission intensity of acceptor, PNAC* (at excitation wavelength $450 \mathrm{~nm}$ ) for different Acceptor/Donor ratios (A:D) in the range from 1:0 to 1:2 in the solution of PNAC* and complementary ssDNA. At 1:1 ratio, the saturation of FRET is observed, there is no further increase in PL emission for higher A: $\mathrm{D}$ ratios.

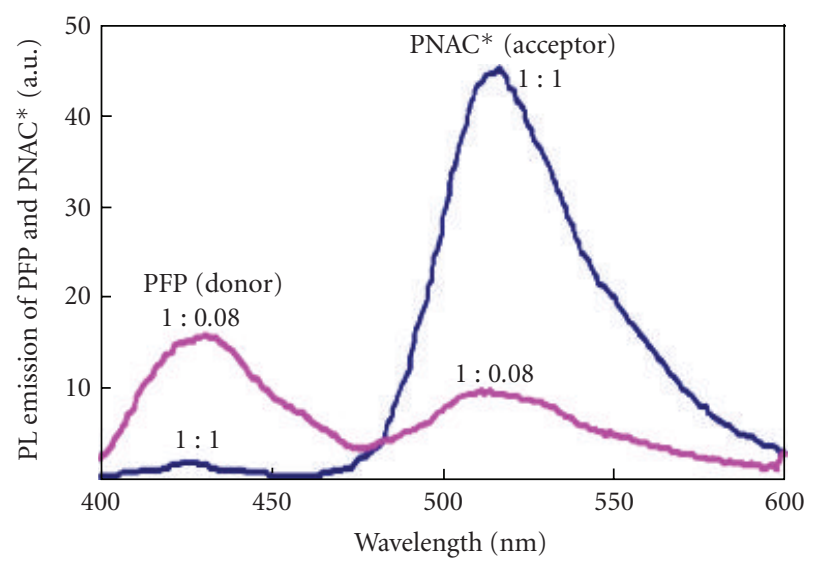

FIgURe 4: PL emission intensity of donor PFP and acceptor PNAC* (at excitation wavelength $450 \mathrm{~nm}$ ) corresponding to A:D ratios, 1:0.08 and 1:1. When FRET is maximum, the donor is quenched by 7 times.

It may be mentioned that we had to use excitation wavelength of $450 \mathrm{~nm}$ to obtain FRET between donor and acceptor. As can be observed from Figure 1, at this wavelength, the absorbance of the donor is reduced by more than $90 \%$ as compared to its peak absorbance. However, as compared to the donor, the acceptor has a very poor absorbance. The peak absorbance of PFP measured by us in the present experiment is 2.8 at $376 \mathrm{~nm}$ and that of PNAC*, 0.016 at $490 \mathrm{~nm}$ and we had to select the excitation wavelength in such a manner that there is sufficient absorbance in the acceptor though at the cost of absorption in the donor.

When the conditions for efficient FRET are satisfied, a significant enhancement in the fluorescence intensity of the acceptor and a decrease in fluorescence intensity of the donor are observed because of the quenching of the fluorescence of the donor as shown in Figure 4.

A comparison of the emission spectra of $\mathrm{PNAC}^{*}$ in the presence of complementary and noncomplementary ssDNA 


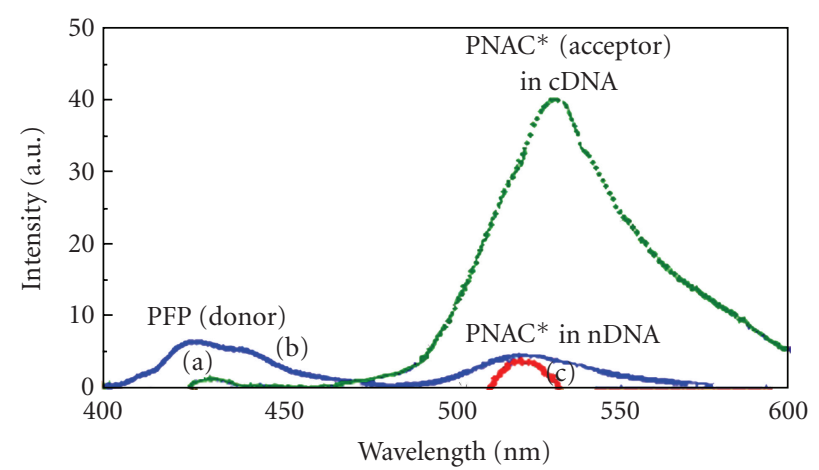

FIgURE 5: PL emission spectra of PFP and PNAC* in presence of complementary (as shown by (a) green colour) and noncomplementary ssDNA (as shown by (b) blue colour). Emission spectra of PNAC* on direct excitation in absence of polymer is also shown (by (c) red colour).

has been shown in Figure 5. Intense Fluorescein emission from fluorescent dye due to efficient FRET was observed in the presence of the complementary DNA strand. The efficiency of FRET is dependent on the inverse sixth power of distance between the donor and acceptor and is given by

$$
E=\frac{1}{\left\{1+\left(R / R_{0}\right)^{6}\right\}},
$$

where $R_{0}$ is the distance at which half of the energy is transferred. The Forster critical radius $R_{0}$ (in nanometers) is related to the spectral overlap by the relation [29]:

$$
R_{0}=2.11 \times 10^{-2}\left[K^{2} \cdot J(\lambda) \cdot \eta^{-4} \cdot Q_{D}\right]^{1 / 6},
$$

where $K^{2}$ is a factor describing the relative orientation in space between the transition dipoles of the donor and acceptor, $J(\lambda)$ is the spectral overlap integral of donor emission and acceptor absorbance (with wavelength in nanometers), $\eta$ is the refractive index of the medium, and $Q_{D}$ is the quantum yield of the donor. If the distance between donor and acceptor is within Forster critical radius $R_{0}$ which is calculated as $6.4 \mathrm{~nm}$ between PFP and Fluorescein using (2), the FRET energy will enhance the acceptor emission and quench the PL of the donor PFP. If the average distance between the hybridized complex and the PFP molecules is more than Forster radius, there will be no efficient transfer of energy from donor to acceptor and hence no emission from the fluorophore. Therefore, this distance has been optimized by varying the concentrations of the donor and acceptor in the solution as shown in Figure 6. We have also calculated the efficiency of energy transfer for different concentrations of donor using (1) and it can be observed from Figure 6 that as the concentration of donor increases, the efficiency of energy transfer also increases till saturation of FRET is achieved and no change was observed beyond the acceptor-donor ratio 1:1, that is, for $1: 3$ and further.

\section{RESULTS AND DISCUSSION}

The condition of maximum overlap between the emission spectrum of the donor PFP and the absorption spectrum

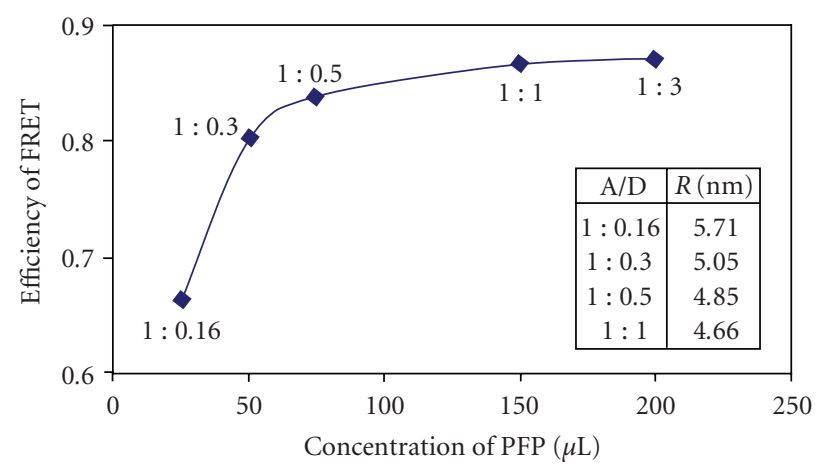

FIGURE 6: Efficiency of energy transfer, that is, FRET from donor to acceptor with the variation in the concentration of PFP. Inset shows the separation distance $R$ between donor and acceptor corresponding to different A:D ratios.

of the acceptor PNAC* for efficient FRET to occur is satisfied as is clear from Figure 2. Fluorescence studies performed for the composite solution PFP/PNAC*/ssDNA (both complementary and noncomplementary at excitation wavelength $450 \mathrm{~nm}$ ) show that the fluorescence emission was about 12 times higher for PFP/PNAC*/ssDNA (complementary) relative to $\mathrm{PFP} / \mathrm{PNAC}^{*} / \mathrm{ssDNA}$ (noncomplementary) complex for which only a weak signal is observed (Figure 5). The experiment was repeated four times to get consistent results within 5\%. This large difference between the emission intensities (for complementary and noncomplementary ssDNA) validates the technique used by us for detection of the DNA sequence where it has been possible to take advantage of the optical amplification of the conjugated polymers to detect DNA hybridization. It can be observed from Figure 6 that the FRET saturates beyond A: D ratio 1:1, and also the separation distance calculated using (1) between donor and acceptor decreases upto this ratio. The technique is not only simple but requires very low $\left(10^{-7}-10^{-8} \mathrm{M}\right)$ concentrations of PFP, PNA, and ssDNA thereby making the sensor cost effective. The reporter emission (PNAC* here) "turns on" only when the target ssDNA is present in the solution. The technique does not require multiple probes and complex DNA structures. It will also be interesting to determine the detection limit of the sensor, that is, the minimum concentration of PNAC* which can be used for the present experiment. We have tried to excite PL in a PNAC* solution having a concentration of $10^{-9} \mathrm{M}$. As will be seen in Figure 7, the instrument does not detect PL emission and only noise is observed. We, therefore, conclude that detection limit of PNAC $^{*}$ is $\sim 10^{-8} \mathrm{M}$.

\section{CONCLUSIONS}

FRET-based sensor for a typical PNA probe for Bacillus anthracis has been tested successfully and presented in the paper. Similarly, PNA probes for various bacteria, such as Escherichia coli, Pseudomonas aeruginosa, and Staphylococcus aureus, will be used to standardize the sensor for other unknown DNA sequences. It may be mentioned that we 


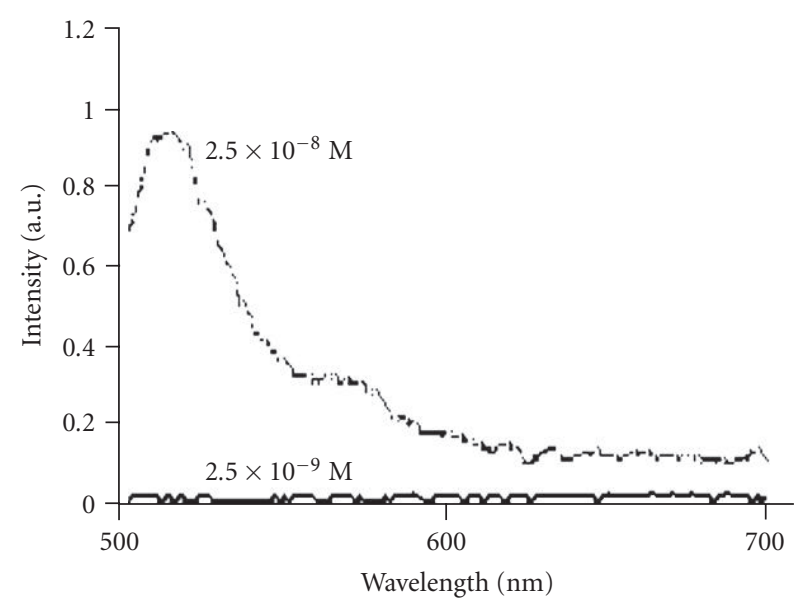

FIgURE 7: PL emission of PNAC* at molar concentration $2.5 \times$ $10^{-8} \mathrm{M}$ and $2.5 \times 10^{-9} \mathrm{M}$ (at excitation wavelength $492 \mathrm{~nm}$ ).

have proposed a low-cost portable sensor [30] based on the above-mentioned technique, where a small UV-Vis lamp is used as the source of exciting the polymer PFP. Under the condition of efficient FRET, the PL from the PFP is quenched, energy is transferred to the dye and significant change in emission intensity of the acceptor is observed. This technique is fast and cost-effective over other conventional diagnostic techniques, which are not only expensive but also timeconsuming and less reliable.

\section{ACKNOWLEDGMENTS}

The authors wish to thank Department of Biotechnology (DBT) INDIA, for the financial support (BT/PR6440/ MED/14/812/2005). Authors also wish to thank Dr. Rani Gupta and Professor R. K Saxena (Department of Microbiology, University of Delhi South Campus, New Delhi, India) for their helpful suggestions and providing help in the experimental work.

\section{REFERENCES}

[1] M. J. Heller, "DNA microarray technology: devices, systems, and applications," Annual Review of Biomedical Engineering, vol. 4, pp. 129-153, 2002.

[2] Y. W. C. Cao, R. Jin, and C. A. Mirkin, "Nanoparticles with Raman spectroscopic fingerprints for DNA and RNA detection," Science, vol. 297, no. 5586, pp. 1536-1540, 2002.

[3] W. Miao and A. J. Bard, "Electrogenerated chemiluminescence. 72. Determination of immobilized DNA and C-reactive protein on $\mathrm{Au}$ (111) electrodes using tris (2,2' - bipyridyl) ruthenium (II) labels," Analytical Chemistry, vol. 75, no. 21, pp. 5825-5834, 2003.

[4] A. W. Peterson, L. K. Wolf, and R. M. Georgiadis, "Hybridization of mismatched or partially matched DNA at surfaces," Journal of the American Chemical Society, vol. 124, no. 49, pp. 14601-14607, 2002.

[5] X. Wang, K. L. Cooper, A. Wang, et al., "Label-free DNA sequence detection using oligonucleotide functionalized optical fiber," Applied Physics Letters, vol. 89, no. 16, Article ID 163901, 3 pages, 2006.
[6] N. Kumar, A. Dorfman, and J.-I. Hahm, "Ultrasensitive DNA sequence detection using nanoscale $\mathrm{ZnO}$ sensor arrays," Nanotechnology, vol. 17, no. 12, pp. 2875-2881, 2006.

[7] M. A. Cooper, F. N. Dultsev, T. Minson, V. P. Ostanin, C. Abell, and D. Klenerman, "Direct and sensitive detection of a human virus by rupture event scanning," Nature Biotechnology, vol. 19, no. 9, pp. 833-837, 2001.

[8] F. Höök, A. Ray, B. Nordén, and B. Kasemo, "Characterization of PNA and DNA immobilization and subsequent hybridization with DNA using acoustic-shear-wave attenuation measurements," Langmuir, vol. 17, no. 26, pp. 8305-8312, 2001.

[9] C. Fan, K. W. Plaxco, and A. J. Heeger, "Electrochemical interrogation of conformational changes as a reagentless method for the sequence-specific detection of DNA," Proceedings of the National Academy of Sciences of the United States of America, vol. 100, no. 16, pp. 9134-9137, 2003.

[10] E. M. Boon, D. M. Ceres, T. G. Drummond, M. G. Hill, and J. K. Barton, "Mutation detection by electrocatalysis at DNAmodified electrodes," Nature Biotechnology, vol. 18, no. 10, pp. 1096-1100, 2000.

[11] J. Fritz, E. B. Cooper, S. Gaudet, P. K. Sorger, and S. R. Manalis, "Electronic detection of DNA by its intrinsic molecular charge," Proceedings of the National Academy of Sciences of the United States of America, vol. 99, no. 22, pp. 14142-14146, 2002.

[12] F. Patolsky, A. Lichtenstein, and I. Willner, "Detection of single-base DNA mutations by enzyme-amplified electronic transduction," Nature Biotechnology, vol. 19, no. 3, pp. 253257, 2001.

[13] C. J. Yu, Y. Wan, H. Yowanto, et al., "Electronic detection of single-base mismatches in DNA with ferrocene-modified probes," Journal of the American Chemical Society, vol. 123, no. 45, pp. 11155-11161, 2001.

[14] E. A. Winzeler, M. Schena, and R. W. Davis, "Fluorescencebased expression monitoring using microarrays," Methods in Enzymology, vol. 306, pp. 3-6, 1999.

[15] A. J. Heeger, "Nobel lecture: semiconducting and metallic polymers: the fourth generation of polymeric materials," Reviews of Modern Physics, vol. 73, no. 3, pp. 681-700, 2001.

[16] L. Chen, D. W. McBranch, H.-L. Wang, R. Helgeson, F. Wudl, and D. G. Whitten, "Highly sensitive biological and chemical sensors based on reversible fluorescence quenching in a conjugated polymer," Proceedings of the National Academy of Sciences of the United States of America, vol. 96, no. 22, pp. 12287-12292, 1999.

[17] C. Fan, K. W. Plaxco, and A. J. Heeger, "High-efficiency fluorescence quenching of conjugated polymers by proteins," Journal of the American Chemical Society, vol. 124, no. 20, pp. 5642-5643, 2002.

[18] P. S. Heeger and A. J. Heeger, "Making sense of polymer-based biosensors," Proceedings of the National Academy of Sciences of the United States of America, vol. 96, no. 22, pp. 12219-12221, 1999.

[19] C. Fan, S. Wang, J. W. Hong, G. C. Bazan, K. W. Plaxco, and A. J. Heeger, "Beyond superquenching: hyper-efficient energy transfer from conjugated polymers to gold nanoparticles," Proceedings of the National Academy of Sciences of the United States of America, vol. 100, no. 11, pp. 6297-6301, 2003.

[20] T. M. Swager, "The molecular wire approach to sensory signal amplification," Accounts of Chemical Research, vol. 31, no. 5, pp. 201-207, 1998.

[21] T. M. Swager and J. H. Wosnick, "Self-amplifying semiconducting polymers for chemical sensors," MRS Bulletin, vol. 27, no. 6, pp. 446-450, 2002. 
[22] M. Egholm, O. Buchardt, L. Christensen, et al., "PNA hybridizes to complementary oligonucleotides obeying the Watson-Crick hydrogen-bonding rules," Nature, vol. 365, no. 6446, pp. 566-568, 1993.

[23] P. E. Nielsen, "Applications of peptide nucleic acids," Current Opinion in Biotechnology, vol. 10, no. 1, pp. 71-75, 1999.

[24] V. V. Demidov, V. N. Potaman, M. D. Frank-Kamenetskil, et al., "Stability of peptide nucleic acids in human serum and cellular extracts," Biochemical Pharmacology, vol. 48, no. 6, pp. 1310-1313, 1994.

[25] J. R. Lakowicz, Principles of Fluorescence Spectroscopy, Springer, New York, NY, USA, 2006.

[26] A. Dietrich, V. Buschmann, C. Müller, and M. Sauer, "Fluorescence resonance energy transfer (FRET) and competing processes in donor-acceptor substituted DNA strands: a comparative study of ensemble and single-molecule data," Reviews in Molecular Biotechnology, vol. 82, no. 3, pp. 211-231, 2002.

[27] Q.-H. Xu, S. Wang, D. Korystov, et al., "The fluorescence resonance energy transfer (FRET) gate: a time-resolved study," Proceedings of the National Academy of Sciences of the United States of America, vol. 102, no. 3, pp. 530-535, 2005.

[28] B. S. Gaylord, A. J. Heeger, and G. C. Bazan, "DNA detection using water-soluble conjugated polymers and peptide nucleic acid probes," Proceedings of the National Academy of Sciences of the United States of America, vol. 99, no. 17, pp. 10954-10957, 2002.

[29] Fluorescent Resonance Energy Transfer (FRET) as a probe of proximity in proteins, http://dwb.unl.edu/Teacher/NSF/C08/ C08Links/pps99.cryst.bbk.ac.uk/projects/gmocz/fret.htm.

[30] P. K. Bhatnagar, P. C. Mathur, N. Mathur, and A. Aneja, "Low cost portable sensors for medical diagnostics using Forster resonance energy transfer between water soluble cationic conjugated polymers and PNAC* probes," in Proceedings of the 18th International Conference on Materials for Advanced Technologies (ICMAT'07), p. 26, Singapore, July 2007. 

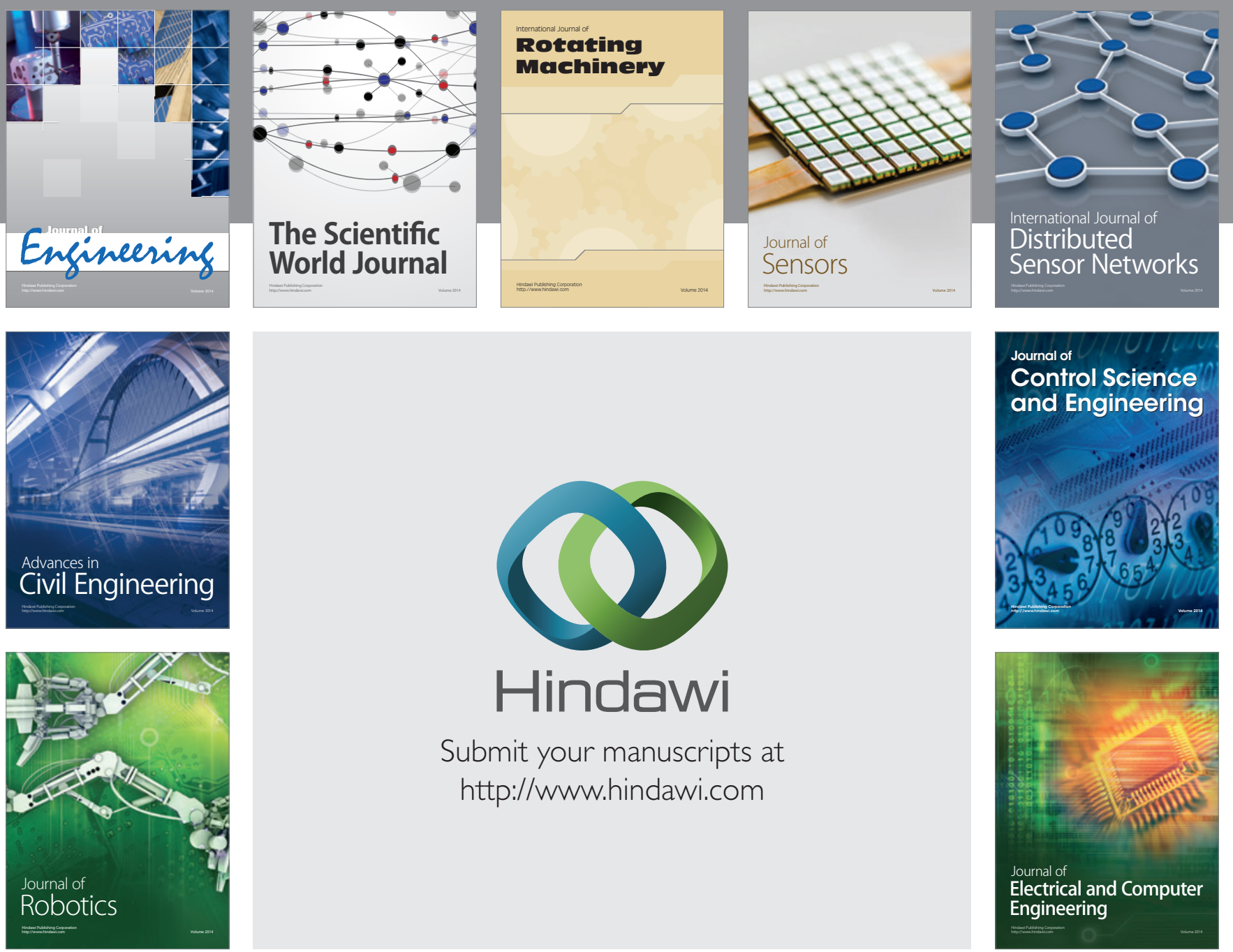

Submit your manuscripts at

http://www.hindawi.com
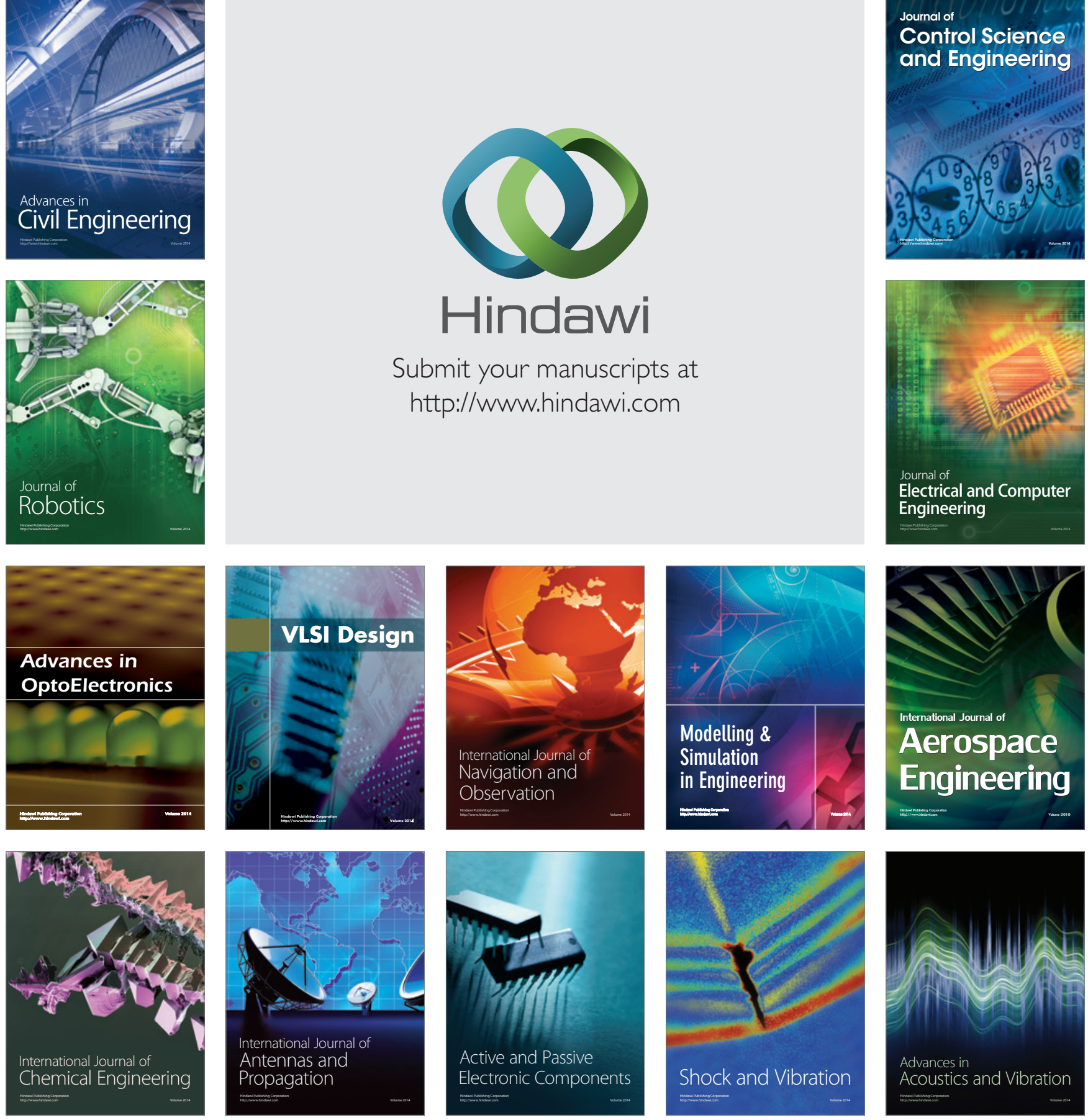